\title{
The Galactic electron and positron "excesses": astrophysical versus dark matter interpretations
}

\author{
Julien Lavalle*† \\ Dipartimento di Fisica Teorica \& INFN \\ Università di Torino \\ Via Giuria 1 \\ I-10125 Torino - Italia \\ E-mail: lavalledin2p3.fr
}

\begin{abstract}
Measurements of a rise in the cosmic positron fraction up to $\sim 100 \mathrm{GeV}$ by the PAMELA satellite have triggered putative interpretation attempts in terms of dark matter (DM) annihilation or decay, even though generic DM particle models are not expected to significantly contribute to this signal. Here, we review the tricks that were invoked to make DM-induced signals fit the data, and discuss their relevance. We also show that considering conventional astrophysical sources - supernova remnants (SNRs) and pulsars - in a consistent cosmic-ray (CR) propagation framework can easily explain these observations, despite large theoretical uncertainties. This does not dismiss potential DM contributions, but this makes the related background far too difficult to control for discovery purposes. Anyway, although the positron puzzle appears qualitatively solved in terms of standard astrophysics, substantial improvements in the CR source and propagation modelings are now clearly necessary to improve our understanding of the Galactic CR lepton budget and induced multi-wavelength diffuse emissions, still relevant to DM searches.
\end{abstract}

Identification of Dark Matter 2010-IDM2010

July 26-30, 2010

Montpellier France

\footnotetext{
* Speaker.

${ }^{\dagger}$ Current address: Department and Institute of Theoretical Physics, Autonomous University of Madrid — Spain
} 


\section{Introduction}

Indications of an increase in the $\mathrm{CR}$ positron fraction above a few $\mathrm{GeV}$ have been collected for quite a long time [1-4], but the recent PAMELA measurements have reached an unprecedented statistics allowing much more detailed analyses [5]. This increase can hardly be explained in terms of secondary positrons ${ }^{1}[6,7]$, though peculiar spatial effects are still worth being investigated into more details [8] — see also the original proposal by [9] about a possible solar magnetic lens effects.

Antimatter CRs as potential probes of DM annihilation were first proposed in [10] after one became aware of the power of gamma-ray and CR observations to constrain the DM properties (e.g. [11-13]). Later, as already mentioned in [14], the authors of [15] showed that the annihilation of supersymmetric neutralinos, the most popular DM species candidate so far, could hardly generate observable features in the local positron spectrum unless the annihilation rate is substantially boosted, stressing that DM substructures could play this amplifier role, as originally suggested in [16]. It was recently shown, however, that such subhalos can actually hardly be at the origin of the large required enhancement $[17,18]$, and that even an isolated such dark source, as proposed in [19], would lead to tensions with current gamma-ray constraints [20]. Despite this apparent failure of DM particle models to naturally yield CR positrons in sufficient amount, an impressive number of papers has been released in the past few years to try to explain the PAMELA data in terms of DM annihilation or decay. We will discuss a few aspects of the available proposals below, but it is already worth noticing that all these works assumed the absence of astrophysical sources of positrons but secondaries when fitting the data.

Although interpretations in terms of standard astrophysical sources were only a few in the past decades (e.g. [21-25]), they all relied on quite sound physical arguments: pulsars were already predicted to produce a significant amount of electron-positron pairs only few years after their discovery (e.g. [26]), which is now indirectly proven from the more recent observations of gamma-rays originating from pulsar magnetospheres. Another way to generate primary-like positrons, also relying on standard astrophysics and recently widely surveyed, is to invoke the diffusive acceleration of those secondary positrons created at SNR shocks (e.g. [27, 28]). Nevertheless, we will focus here on the pulsar solution when discussing standard astrophysical explanations of the positron excess.

The outline of this proceeding is the following. We first recall the bases of electron-positron propagation in the Galaxy. Then, we revisit the case for the DM interpretation of the cosmic positron excess, showing how contrived this attempt can be. Afterwards, we discuss the requirements a model of astrophysical CR electrons and positrons should obey. We emphasize that including pulsars as positron sources can naturally lead to a good fit of the PAMELA data, with quite reasonable parameters. Finally, we conclude and discuss a few perspectives.

\section{Bases for cosmic-ray propagation}

Reviews and books on CR propagation are numerous, and we refer the reader to e.g. [29] for a recent review, and to [30] and [31, 32] for valuable books. A detailed description of CR electron ${ }^{2}$

\footnotetext{
${ }^{1}$ For the non-expert reader, a primary cosmic ray is produced — or/and accelerated — at sources, whereas a secondary cosmic ray originates from nuclear interactions between primary CR nuclei and the interstellar gas (ISG).
} 
propagation can be found in [33].

Once produced, stable charged CRs (among which electrons) in the $\mathrm{GeV}-\mathrm{TeV}$ energy range may experience different processes. The dominant ones are diffusion in space (due to scattering with magnetic turbulences and to convective winds) and diffusion in momentum (energy losses negligible for $\mathrm{CR}$ nuclei — and diffusive reacceleration — negligible above a few $\mathrm{GeV}$ ). Electron propagation in this energy range is almost completely set by spatial diffusion and energy losses, the latter being mainly due to inverse Compton interactions with the interstellar radiation field (ISRF, including the CMB) and the Galactic magnetic field (synchrotron). In steady state and when convection is neglected, the master propagation equation associated with an electron density $\mathscr{N} \equiv d n / d E$ reads

$$
-\vec{\nabla}\{K(E, \vec{x}) \vec{\nabla} \mathscr{N}(E, \vec{x})\}-\partial_{E}\{b(E) \mathscr{N}(E, \vec{x})\}=\mathscr{Q}(E, \vec{x}),
$$

where $\mathscr{Q}$ is the source term, $K(E, \vec{x})$ is the diffusion coefficient, and $b(E)=-d E / d t$ is the energy loss rate; being set by Compton interactions, the latter strongly increases with energy $\left(b(E) \propto E^{2}\right.$ in the Thomson approximation). The typical energy loss timescale is $\tau_{l} \approx 10^{16} \mathrm{~s}$ at $1 \mathrm{GeV}$ for Galactic electrons. Note that the diffusion coefficient can, in most of relevant situations, be considered as homogeneous, and is usually modeled as a power law, $K(E) \simeq K_{0}\left(E / E_{0}\right)^{\delta}$. The normalization and index can be determined locally from observed secondary-to-primary ratios of nuclei species which mostly depend on $K_{0} / L$ in a slab diffusion model, where $L$ is the half-thickness of the diffusion zone (see e.g. $[34,35])$ - values are usually found close to $K_{0}\left(E_{0}=1 \mathrm{GeV}\right) \approx 3.4 \times 10^{27} \mathrm{~cm}^{2} / \mathrm{s}$, $\delta \approx 0.7$ and $L \approx 4 \mathrm{kpc}$. The above equation (without spatial boundaries) admits a solution in terms of a Green function

$$
\mathscr{G}\left(E, \vec{x} \leftarrow E_{s}, \vec{x}_{s}\right)=\frac{\exp \left\{-\frac{\left(\vec{x}_{s}-\vec{x}\right)^{2}}{\lambda^{2}}\right\}}{b(E)\left(\pi \lambda^{2}\right)^{\frac{3}{2}}} \text { with } \lambda^{2} \equiv 4 \int_{E}^{E_{s}} d E^{\prime} \frac{K\left(E^{\prime}\right)}{b\left(E^{\prime}\right)},
$$

where $\lambda$ is the mean propagation scale, of the order of $\sqrt{K_{0} \tau_{l}} \approx 2 \mathrm{kpc}$, i.e. usually less than $L$ (spatial boundaries can in most of cases be neglected to a good approximation): due to the very efficient energy losses, electrons with energies above a few tens of $\mathrm{GeV}$ are mostly local. This Green function allows the computation of the electron density at the Earth given a source term $\mathscr{Q}\left(E_{s}, \vec{x}_{s}\right)$ which features the spatial and energy distributions before propagation.

Primary and secondary CRs of astrophysical origin are injected in the Galactic plane, where the standard sources (SNRs and pulsars) and the ISG are located. For secondary positrons, which will constitute the "background" in the next sections of this proceeding, one can approximate the source term as a power law spectrum of index $\gamma_{s}$, reminiscent from the CR nuclei index, and flatly distributed in the Galactic plane, such that $\mathscr{Q}_{s} \propto E^{-\gamma_{s}} \delta(z)$. Thus (in the Thomson approximation for the energy losses), the secondary positron flux can be derived analytically: $\phi_{s} \propto \sqrt{\tau_{l} / K_{0}} E^{-\tilde{\gamma}_{s}}$, where $\tilde{\gamma}_{s}=\gamma_{s}+0.5(\alpha+\delta-1)-\alpha \equiv \ln \left(b(E) / b\left(E_{0}\right)\right) / \ln \left(E / E_{0}\right)$, i.e. $\alpha=2$ in the Thomson approximation. Note that this reasoning also holds for high energy primaries when neglecting the local discreteness of the source distribution. By using the index inferred from considering CR nuclei interactions with the ISG, i.e. $\gamma_{s} \simeq 2.75$, one readily gets $\tilde{\gamma}_{s} \simeq 3.45$, quite close to the observed

\footnotetext{
${ }^{2}$ The term electrons will refer to both electrons and positrons when discussing general propagation features.
} 

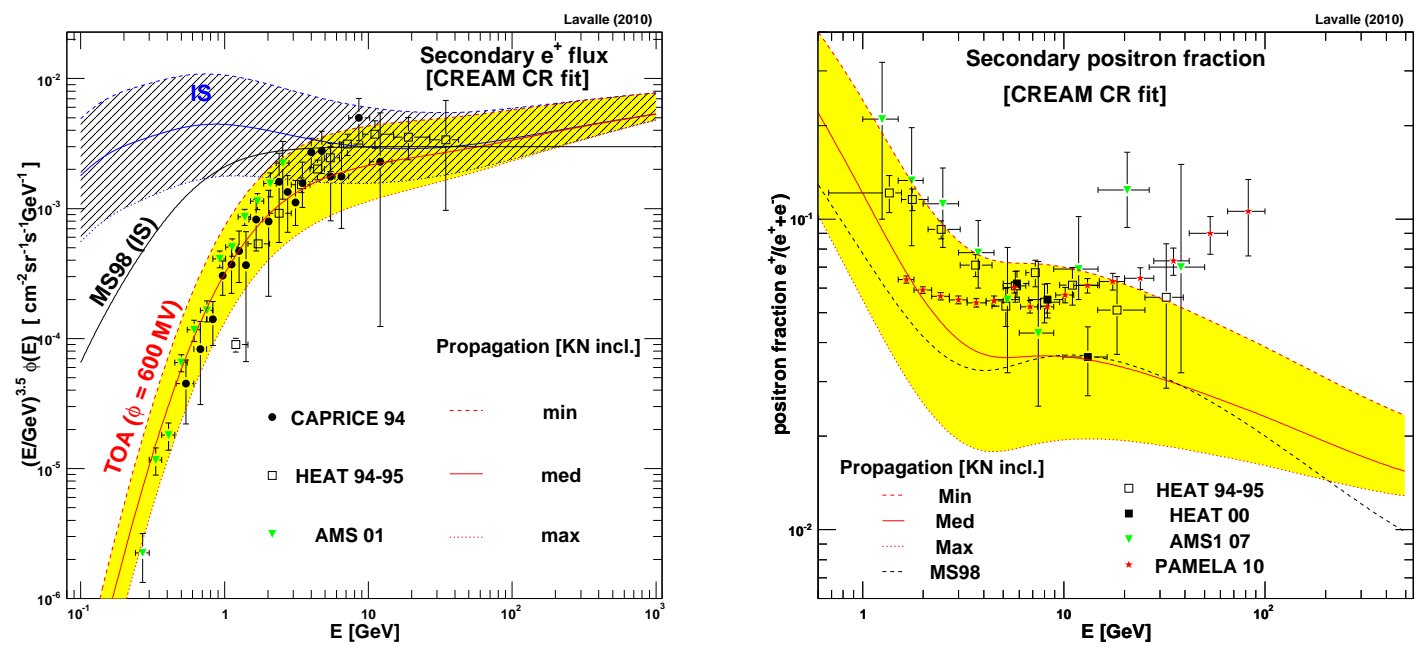

Figure 1: Left: Predictions for the secondary positron flux with a conservative theoretical uncertainty band for the propagation parameters. Right: corresponding positron fraction (taken from [37]). MS98 corresponds to the positron flux prediction calculated in [6].

positron-only index $[7,33]$. Nevertheless, predictions for the secondary positron flux fail to fit the positron fraction data $-f_{e^{+}} \equiv \phi_{e^{+}} /\left(\phi_{e^{-}}+\phi_{e^{+}}\right)$- leading instead to a fraction decreasing with energy [7,33]. Further taking into account the hardening in the proton spectrum above $\sim 2 \mathrm{TeV}$ recently observed by the CREAM experiment [36] results in a slightly harder secondary positron spectrum, but this is obviously not enough to explain the measured increase [37]. Predictions for the secondary positron fraction including this effect are displayed in Fig. 1, where a conservative range for theoretical uncertainties in the propagation parameters is considered - these predictions can be reproduced from the fitting functions provided in [37].

\section{The dark matter interpretation of the positron signal}

DM particle scenarios rely on theories beyond the standard model. We emphasize that these theories, which were developed to solve problems inherent to particle physics, could simultaneously offer independent and powerful solutions to apparently disconnected issues in particle physics on the one hand (hierarchy problem, unification of forces, etc.), and in astrophysics and cosmology on the other hand (dark matter, leptogenesis, etc.), which is very appealing. In most of related DM scenarios, e.g. in the so-called weakly interacting massive particle (WIMP) paradigm, the total DM abundance is set by annihilation in the early universe (for reviews, see e.g. [40-42]). Originally in thermal and chemical equilibrium with the primordial plasma after inflation, DM chemically decouples when the annihilation rate becomes smaller than the expansion rate of the universe. This usually has to happen before big bang nucleosynthesis (BBN), which must not be unsettled, when WIMPs are already non-relativistic — referred to as cold DM (CDM). Therefore, cosmology imposes strong constraints to the annihilation cross section, provided the expansion rate of the universe before BBN is taken standard ${ }^{3}$. Typically, to get a relic abundance of $\Omega_{\mathrm{DM}} \approx 0.1 / h^{2}$, WIMPs 

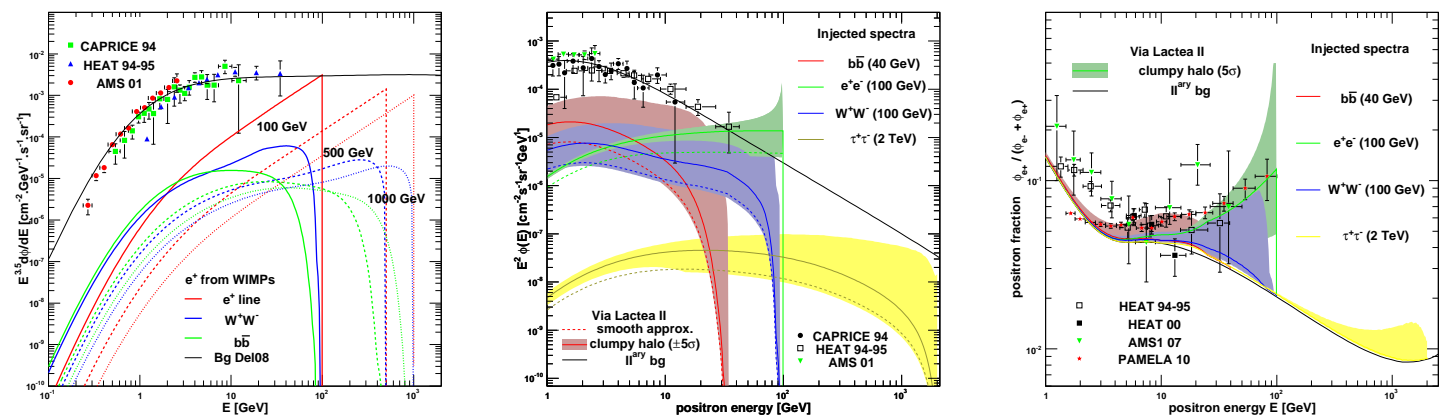

Figure 2: Left: generic predictions for the positron flux associated with a thermal WIMP annihilating into different final states (taken from [38]). Middle: positron flux for a few benchmark models and uncertainty band coming from the effect of subhalos (taken from [39]). Right: positron fractions corresponding to the middle panel flux predictions.

need a thermally averaged annihilation cross section of $\langle\sigma v\rangle \approx 3 \times 10^{-26} \mathrm{~cm}^{3} / \mathrm{s}$.

Annihilation is revived after DM has collapsed to form galaxies, its density being large enough in these objects. WIMPs are now expected to annihilate almost at rest and produce pairs of standard model particles, some of them further hadronizing or decaying, usually leading to the injection of CRs with continuous energy spectra. Attempts to detect these annihilation products refer to as indirect detection (see e.g. $[44,45]$ ). The knowledge of the DM density distribution is crucial to compute the induced CR fluxes, since the annihilation rate scales like the squared density; it is generally expressed in terms of the ratio of the mass density profile $\rho$ to the particle mass $m_{\chi}, n_{\chi}=$ $\rho / m_{\chi}$, and constrained theoretically from $\mathrm{N}$-body simulations and observationally from kinematic data (e.g. [46]).

In the case of high energy positrons, which are short range CRs, the most relevant input is the local DM density set by $\rho_{\odot} \approx 0.3 \mathrm{GeV} / \mathrm{cm}^{3}$. At sufficiently high energy, positrons lose their energy before they substantially diffuse, so that one can neglect spatial diffusion to a good approximation, provided the injection rate does not fluctuate too much over short distances. In that case, the Green function defined earlier becomes $\mathscr{G}\left(E, \vec{x} \leftarrow E_{s}, \vec{x}_{s}\right) \stackrel{\lambda \rightarrow_{0}^{0}}{\approx} \delta^{3}\left(\vec{x}_{s}-\vec{x}\right) / b(E)$. The positron flux at the Earth generated by DM annihilation is thus completely analytical:

$$
\begin{aligned}
\phi_{e^{+}}(E) & \approx \frac{\beta c}{4 \pi} \frac{\langle\sigma v\rangle}{2 b(E)}\left[\frac{\rho_{\odot}}{m_{\chi}}\right]^{2} \int_{E}^{m_{\chi}} d E_{s} \frac{d N_{e^{+}}}{d E_{s}} \\
& \approx \phi_{\chi}^{0}\left[\frac{\langle\sigma v\rangle}{3 \times 10^{-26} \mathrm{~cm}^{3} / \mathrm{s}}\right]\left[\frac{\tau_{l}}{10^{16_{\mathrm{s}}}}\right]\left[\frac{\rho_{\odot} /\left(0.3 \mathrm{GeV} / \mathrm{cm}^{3}\right)}{(E / 1 \mathrm{GeV})\left(m_{\chi} / 100 \mathrm{GeV}\right)}\right]^{2} \int_{E}^{m_{\chi}} d E_{s} \frac{d N_{e^{+}}}{d E_{s}},
\end{aligned}
$$

where we find $\phi_{\chi}^{0}=3.2 \times 10^{-6} \mathrm{~cm}^{-2} \mathrm{~s}^{-1} \mathrm{GeV}^{-1} \mathrm{sr}^{-1}$ - if WIMPs are Dirac fermions, an additional factor of $1 / 2$ must be accounted for. Notice the dependence on energy and WIMP mass, which is explicit in this equation. We can further simplify it by assuming annihilation into electronpositron pairs, so that the injected spectrum $d N_{e^{+}} / d E_{s}=\delta\left(E_{s}-m_{\chi}\right)$. In this case, the positron flux associated with a WIMP mass of $m_{\chi}=100 \mathrm{GeV}$ at an energy of $100 \mathrm{GeV}$ is $\phi_{\chi}\left(E=m_{\chi}=\right.$

\footnotetext{
${ }^{3}$ This could change e.g. in the context of quintessence as a solution to dark energy (e.g. [43]).
} 
$100 \mathrm{GeV}) \approx 3.2 \times 10^{-10} \mathrm{~cm}^{-2} \mathrm{~s}^{-1} \mathrm{GeV}^{-1} \mathrm{sr}^{-1}$, amazingly close to the value predicted for the secondary background at this energy. This means that such a model, provided a small amplification (or boost) by a factor of a few, could very well explain the observed rise in the positron fraction [39] (see right panel of Fig. 2). Nevertheless, annihilation exclusively into lepton pairs is quite a contrived case for WIMPs with masses greater than a few $\mathrm{GeV}$, which can neither rely on sound particle physics motivations nor be easily cooked up. For other annihilation final states, like heavy quarks or massive gauge bosons, the required boost factor is much larger - see e.g. [38] and the left panel of Fig. 2. Since the annihilation cross section is fixed by the relic abundance, this feeble positron flux is a quite generic prediction, valid for most of motivated DM particle scenarios like in supersymmetry or extra-dimensions [47].

At this stage, the question is: are there ways to amplify the predicted signal which would be motivated by some solid physical arguments? Basically, there are three different directions we can try: (i) enhancing the annihilation cross section; (ii) playing with CR propagation: theoretical uncertainties allow some freedom; (iii) considering extra (local) sources of DM. For DM decay scenarios, which we will not discuss further here, we note that the decay rate strongly suffers arbitrariness, which makes questionable the relevance of such models as natural explanations to the PAMELA signal; likewise, those that fit the PAMELA data are in turn quite in tension with gamma-ray observations [48].

Enhanced annihilation cross section: The physical motivation behind this possibility is that when WIMPs are much more massive than an exchanged virtual force carrier during the annihilation process, then non-perturbative effects can strongly amplify the cross section and even make resonances arise (referred to as Sommerfeld enhancement [49, 50]). At resonances, this effect scales like the inverse squared WIMP velocity, so it is much stronger in galaxies today than at the decoupling time in the early universe. Nevertheless, this effect concerns a very small part of the WIMP parameter space, and suffers severe constraints: beside inherent limitations initially neglected [51], all signals associated with the amplified final state are enhanced the same, which generally leads the predicted antiproton flux to overshoot the observational bounds, unless heavy DM particles annihilate only into leptons, which is quite contrived [52]. Such a possibility has therefore poor relevance. Incidentally, note that current antiproton data are also quite constraining for low WIMP masses [53].

Impact of theoretical uncertainties in CR propagation: Since DM-induced CRs are produced everywhere in the Galaxy, enlarging the diffusion zone in the range permitted by theoretical uncertainties may increase the flux predictions. Nevertheless, for positrons, this would only affect the low energy part of the spectrum, since high energy positrons, bound to be short range by efficient energy losses, must originate from very local regions. Therefore, tuning the propagation parameters also fails to enhance the high energy positron flux $[18,54]$ — the energy loss parameters being quite well constrained.

Considering extra DM sources: The impact of DM substructures wandering in the Galactic halo was first emphasized by [16] (see also [55] for more insights on their connection with the WIMP properties). Nevertheless, predicting the positron boost factor associated with these subhalos is not trivial, since it depends on both their inner properties and their spatial distribution [17]. Even when spanning the full ranges for prescriptions coming from cosmological structure formation theory, it was actually shown that these Galactic subhalos could not increase the signal by 

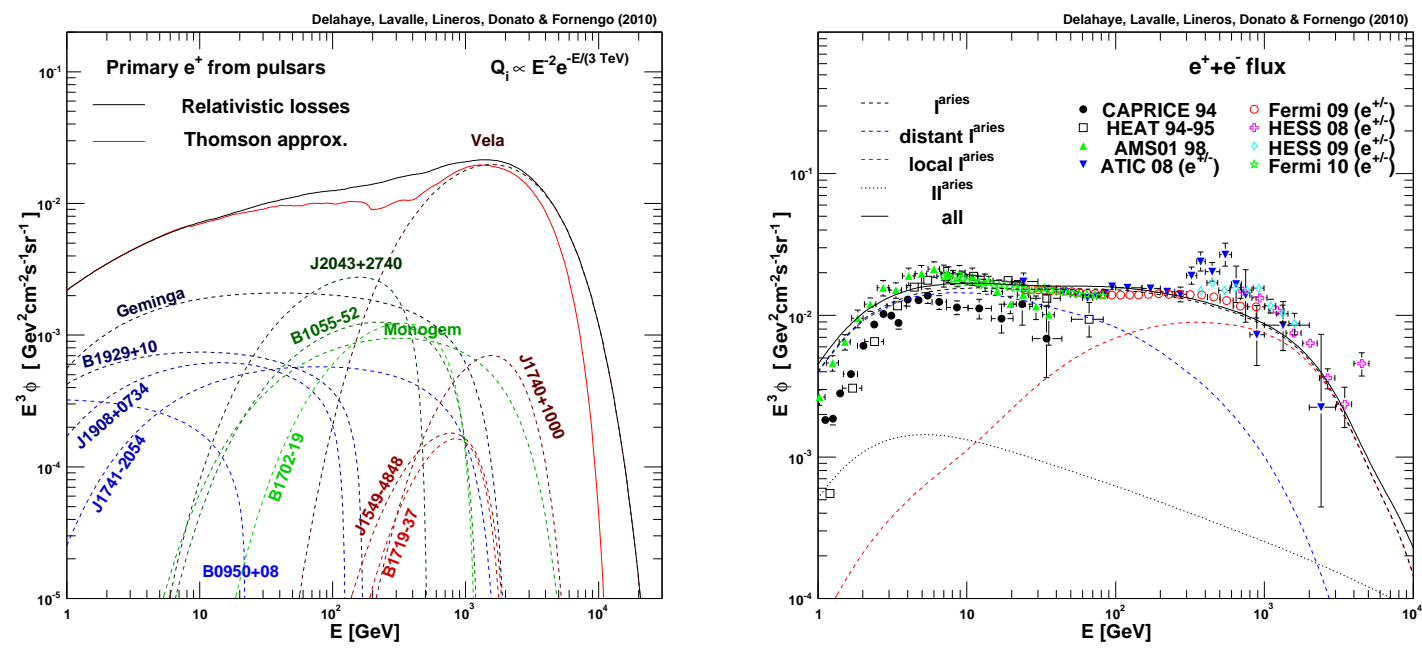

Figure 3: Left: contribution of local pulsars to the positron flux. Right: example of a self-consistent prediction for both electrons and positrons. These plots are borrowed from [33].

a large factor, with an upper limit $\lesssim 20$ [18]. However, this upper bound is associated with a large statistical variance at high energy reflecting the fact that though the probability of finding a massive enough subhalo close to the Earth is vanishingly small, this would amplify the signal by a larger factor if this occurred. Nevertheless, even this tricky situation suffers strong constraints coming e.g. from gamma-ray observations, which strongly disfavor it as an explanation to a rising positron fraction [20]. Up-to-date predictions of the DM-induced positron flux including the effect of subhalos have been recently released in [39] and are displayed in Fig. 2; they show, as discussed above, that subhalos can hardly rehabilitate thermal WIMPs as a satisfactory explanation to the positron excess, except in the poorly motivated case of an annihilation into light charged lepton pairs.

To conclude this section, we emphasize that the DM solution to the cosmic positron issue is by itself very contrived and lacks strong physical motivations. Likewise, most of attempts are now excluded by complementary constraints coming from other cosmic messengers. Finally, note that all predictions implying DM assume the absence of other astrophysical sources of positrons, which is incorrect. As explained in the next section, the actual astrophysical background relevant to DM searches in the positron channel is in fact quite far from controled because of this primary component that remains to be fully characterized, which unfortunately makes this channel inappropriate to DM discovery purposes, at least temporarily.

\section{The astrophysical solution: towards a self-consistent cosmic-ray lepton model}

Pulsars have long been proposed as sources of CR positrons, and were even demonstrated, more than 15 years ago, to provide a good fit to the observed positron fraction above $5 \mathrm{GeV}$. Since there are also other astrophysical proposals beside pulsars that could provide additional positrons, the question is not really whether standard astrophysics can explain the data, but what a consistent 
CR model should look like - note that the whole set of data such a model has to fit must also include the individual spectra of electrons and positrons, and their sum as recently measured by Fermi and HESS up to TeV energies [56, 57]. In fact, it is probably because it was wrongly believed that a standard model of CRs existed that PAMELA measurements triggered such a "buzz". Indeed, the most popular numerical CR model called GALPROP [58, 6], which was too naively taken as a reference in the debate on positrons, (i) was not including any source of primary positrons and (ii) was treating the injection of electrons with a smooth and continuous spatial distribution of SNRs associated with an empirical energy spectrum, which also led to the claim of an excess in the Fermi data. Aside from the lack of primary positrons in this model, it has indeed been known for long that considering a smooth spatial distribution of sources failed for local high energy flux predictions: the true discreteness of local sources must induce spectral fluctuations in the high energy data [59,60]. Yet, this confusion cannot be attributed to the GALPROP model itself, since it was originally more dedicated to the study of the diffuse Galactic multi-wavelength emissions originating from CR interactions with the interstellar medium (ISM), either hadronic and electromagnetic, than to the local electron budget.

Therefore, while we can fairly talk about a standard paradigm for CR propagation and sources, a standard model is still far from achieved. A consistent prediction of the CR electron and positron fluxes at the Earth (or at any point in the Galaxy) should at least include the contributions of (i) secondaries, (ii) a smooth distribution of sources for the distant (and therefore low energy) primary component, with a radial cut-off set to a distance from the Earth for which the source discreteness effects can safely be averaged out ( $\sim 2 \mathrm{kpc}$ ), and (iii) the discrete sources located inside this cut-off radius. For the latter contribution, different catalogs are available for pulsars and SNRs to constrain the relevant parameters, e.g. the position and age estimates [61, 62]. It is clear that for a given luminosity, the more distant the source, the more difficult to observe. This means that the above approach will be more reliable in predicting the local high energy electron density than in predicting it in more distant regions. In any case, we emphasize that for point sources, Klein-Nishina effects must be included in the treatment of energy losses to derive reliable predictions [60, 33].

Beside consistency in the spatial distribution of sources and propagation, there is still a major issue to mention: the injection of electrons from SNRs and pulsars. Indeed, though we can reasonably motivate an averaged power law spectrum associated with an injection rate set by the explosion rate of supernovæ and some energetics considerations for the distant component [63], an accurate modeling of local sources is of paramount importance, since few of them might dominate the overall flux above $\sim 100 \mathrm{GeV}$ : the overall spectrum piles up all individual contributions and likely departs from a simple power law at high energy. Individual amplitudes and spectral shapes strongly depend not only on the injected spectra, but also on the source distances and ages [33]. A big uncertainty concerns the dynamics of CR injection into the ISM (see e.g. [64, 65]). Nevertheless, this drawback is fairly compensated by the fact that for local sources, we have at hand a numerous multi-wavelength observations to build a rather constrained model for each of them (e.g. [66]). Although a thorough study involving sophisticated modelings for both local pulsars and SNRs remains to be achieved, some preliminary efforts have already been undergone in this direction. In [33], we have notably demonstrated that considering the observational properties of local sources is sufficient to fit the whole set of data on CR electrons and positrons without overtuning the parameters (e.g. invoking very hard spectral indices, etc.) - see Fig. 3. In this study, few sources 
are shown to dominate the high energy $e^{+} e^{-}$budget, as expected. This means that purely stochastic approaches which do not include the known local sources are likely strongly overestimating the actual uncertainties in the expected local lepton flux ${ }^{4}$, and that, in contrast, other approaches based on refining the observed sources modeling are certainly more promising. This paves the road to further developments.

\section{Conclusion}

We have discussed the DM interpretation of the so-called electron and positron excesses, showing that it was likely too contrived to be supported from reasonable grounds, though one cannot formally exclude a DM contribution to the electron and positron spectra. We have also emphasized that consistently considering well known positron sources like pulsars can easily explain the current available data, despite the still large theoretical errors associated with the predictions. This not only means that the explanation to these excesses is no longer an issue, but also, unfortunately, that the positron channel is no longer an interesting discovery channel for indirect DM detection. Indeed, the uncertainties affecting the predictions make the background relevant to DM searches very hard to control.

Nevertheless, these new measurements of high energy CR electrons allow us to refine the current models of $\mathrm{CR}$ propagation and sources. Beside studying more sophisticated propagation treatments (e.g. [67]), improving and testing the physics of CR injection from sources is likely an important topic for the next few years, which can now be implemented thanks to these unprecedented new data. Finally, clarifying our understanding of local CR electrons will have a paramount impact on the predictions for associated multi-wavelength electromagnetic diffuse emissions not only on the Galactic scale, but also beyond (see e.g. the impact of electron propagation on the prediction of the Sunyaev-Zel'dovich effect associated with DM in galaxy clusters or Galactic subhalos in $[68,69])$ - note that studies on diffuse Galactic emissions are very important for indirect DM detection. Among other interesting topics, this may help developing novel approaches to study the Galactic magnetic fields through the synchrotron emission, and also the diffuse gamma-ray emission in the central regions of the Galaxy, the data analysis of which will be soon released by the Fermi collaboration. In this respect, it is clear that the forthcoming lower frequency data expected from instruments like Planck or Herschel $[70,71]$ will be valuable in this research field, as well as higher energy data on local electrons expected from AMS- $02^{5}$.

Acknowledgements: I am grateful to the organizers for inviting me to give this plenary talk, also for their welcome and the impressive and delicious cocktail dinatoire de bienvenue they arranged in the courtyard of Montpellier's historical Faculty of Medicine. Furthermore, it is a pleasure to thank T. Delahaye and R. Lineros for active collaboration and stimulating discussions on the topic.

\footnotetext{
${ }^{4}$ Some Monte Carlo or stochastic approaches are only based on a SN explosion rate and energetic considerations, whereas any random source should be tested as potential observable multi-wavelength emitters, in which case some of them would fall in the contingent of known sources, or would be excluded. Part of the available phase space is therefore already known (observed local sources), an information which is usually not included in these probabilistic treatments.

${ }^{5}$ http: //ams.cern.ch/ http://www.ams02.org/
} 


\section{References}

[1] J. L. Fanselow, R. C. Hartman, R. H. Hildebrad, and P. Meyer, ApJ 158, 771-+, November 1969.

[2] R. L. Golden, B. G. Mauger, S. Horan et al., A\&A 188, 145-154, December 1987.

[3] S. W. Barwick, J. J. Beatty, A. Bhattacharyya et al., ApJL 482, L191+, June 1997.

[4] J. Alcaraz, B. Alpat, G. Ambrosi et al., Phys. Lett. B 484, 10-22, June 2000.

[5] O. Adriani, G. C. Barbarino, G. A. Bazilevskaya et al., Nature 458, 607-609, April 2009.

[6] I. V. Moskalenko and A. W. Strong, ApJ 493, 694-+, January 1998.

[7] T. Delahaye, F. Donato, N. Fornengo, J. Lavalle, R. Lineros, P. Salati, and R. Taillet, $A \& A$ 501, 821-833, July 2009.

[8] N. J. Shaviv, E. Nakar, and T. Piran, Phys. Rev. Lett. 103 (11), 111302-+, September 2009.

[9] J. P. Roberts, ArXiv e-prints, May 2010 [arXiv: 1005 . 4668].

[10] J. Silk and M. Srednicki, Phys. Rev. Lett. 53, 624-627, August 1984.

[11] J. E. Gunn, B. W. Lee, I. Lerche, D. N. Schramm, and G. Steigman, ApJ 223, 1015-1031, August 1978.

[12] F. W. Stecker, ApJ 223, 1032-1036, August 1978.

[13] Ya. B. Zeldovich, A. A. Klypin, M. Yu. Khlopov, and V. M. Chechetkin. Sov. J. Nucl. Phys. 31, 664-669, 1980.

[14] A. J. Tylka, Phys. Rev. Lett. 63, 840-843, August 1989.

[15] E. A. Baltz and J. Edsjö, Phys. Rev. D 59 (2), 023511, January 1998.

[16] J. Silk and A. Stebbins, ApJ 411, 439-449, July 1993.

[17] J. Lavalle, J. Pochon, P. Salati, and R. Taillet, A\&A 462, 827-840, February 2007.

[18] J. Lavalle, Q. Yuan, D. Maurin, and X.-J. Bi, A\&A 479, 427-452, February 2008.

[19] D. Cumberbatch and J. Silk, MNRAS 374, 455-465, January 2007.

[20] T. Bringmann, J. Lavalle, and P. Salati, Phys. Rev. Lett. 103 (16), 161301-+, October 2009.

[21] J. Arons, In G. Setti, G. Spada, \& A. W. Wolfendale, editor, Origin of Cosmic Rays, volume 94 of IAU Symposium, pages 175-204, 1981.

[22] A. K. Harding and R. Ramaty, In International Cosmic Ray Conference, volume 2 of International Cosmic Ray Conference, pages 92-+, 1987.

[23] A. Boulares, ApJ 342, 807-813, July 1989.

[24] F. A. Aharonian, A. M. Atoyan, and H. J. Voelk, A\&A 294, L41-L44, February 1995.

[25] X. Chi, K. S. Cheng, and E. C. M. Young, ApJL 459, L83+, March 1996.

[26] P. A. Sturrock, Nature 227, 465-470, August 1970.

[27] E. G. Berezhko, L. T. Ksenofontov, V. S. Ptuskin, V. N. Zirakashvili, and H. J. Völk, A\&A 410, 189-198, October 2003.

[28] P. Blasi, Phys. Rev. Lett. 103 (5), 051104-+, July 2009. 
[29] A. W. Strong, I. V. Moskalenko, and V. S. Ptuskin, Annual Review of Nuclear and Particle Science, 57, 285-327, November 2007.

[30] V. S. Berezinskii, S. V. Bulanov, V. A. Dogiel, and V. S. Ptuskin, Astrophysics of cosmic rays, Amsterdam: North-Holland, 1990, edited by Ginzburg, V.L., 1990.

[31] M. S. Longair, High energy astrophysics. Vol.1: Particles, photons and their detection, Cambridge, UK: Cambridge University Press, March 1992., March 1992.

[32] M. S. Longair, High energy astrophysics. Vol.2: Stars, the galaxy and the interstellar medium, Cambridge, UK: Cambridge University Press, |c1994, 2nd ed., 1994.

[33] T. Delahaye, J. Lavalle, R. Lineros, F. Donato, and N. Fornengo, $A \& A$ 524, A51, November 2010.

[34] D. Maurin, F. Donato, R. Taillet, and P. Salati, ApJ 555, 585-596, July 2001.

[35] A. Putze, L. Derome, and D. Maurin, A\&A 516, A66+, June 2010.

[36] H. S. Ahn, P. Allison, M. G. Bagliesi et al., ApJL 714, L89-L93, May 2010.

[37] J. Lavalle, ArXiv e-prints, November 2010 [arXiv: 1011.3063$].$

[38] J. Lavalle, In J.-M. Alimi \& A. Fuözfa, editor, AIP Conference Series, volume 1241, pages 398-407, June 2010.

[39] L. Pieri, J. Lavalle, G. Bertone, and E. Branchini, ArXiv e-prints, August 2009 [arXiv:0908.0195].

[40] G. Jungman, M. Kamionkowski, and K. Griest, Phys. Rept. 267, 195-373, March 1996.

[41] L. Bergström, Reports on Progress in Physics 63, 793-841, May 2000.

[42] H. Murayama, ArXiv e-prints, April 2007 [arXiv:0 0704 .2276].

[43] P. Salati, Phys. Lett. B 571, 121-131, October 2003.

[44] J. Carr, G. Lamanna, and J. Lavalle, Reports on Progress in Physics 69, 2475-2512, August 2006.

[45] Pierre Salati. Proceedings of Science, Cargese 2007, 009, 2007.

[46] A. Klypin, H. Zhao, and R. S. Somerville, ApJ 573, 597-613, July 2002.

[47] J. Lavalle, E. Nezri, E. Athanassoula, F.-S. Ling, and R. Teyssier, Phys. Rev. D 78 (10), 103526-+, November 2008.

[48] M. Cirelli, P. Panci, and P. D. Serpico, Nucl. Phys. B 840, 284-303, November 2010.

[49] A. Sommerfeld, Annal Phys. (Leipzig) 403, 257-330, 1931.

[50] J. Hisano, S. Matsumoto, M. M. Nojiri, and O. Saito, Phys. Rev. D 71, 063528-+, March 2005.

[51] J. L. Feng, M. Kaplinghat, and H.-B. Yu, Phys. Rev. D 82, 083525-+, October 2010.

[52] M. Cirelli, M. Kadastik, M. Raidal, and A. Strumia, Nucl. Phys. B 813, 1-2, May 2009.

[53] J. Lavalle, Phys. Rev. D 82 (8), 081302-+, October 2010.

[54] T. Delahaye, R. Lineros, F. Donato, N. Fornengo, and P. Salati, Phys. Rev. D 77 (6), 063527-+, March 2008.

[55] T. Bringmann, New Journal of Physics 11 (10), 105027-+, October 2009.

[56] A. A. Abdo, M. Ackermann, M. Ajello et al., Phys. Rev. Lett., 102 (18), 181101-+, May 2009. 
[57] F. Aharonian, A. G. Akhperjanian, U. Barres de Almeida et al., Phys. Rev. Lett. 101 (26), 261104-+, December 2008.

[58] A. W. Strong and I. V. Moskalenko, ApJ 509, 212-228, December 1998.

[59] C. S. Shen, ApJL 162, L181+, December 1970.

[60] T. Kobayashi, Y. Komori, K. Yoshida, and J. Nishimura, ApJ 601, 340-351, January 2004.

[61] R. N. Manchester, G. B. Hobbs, A. Teoh, and M. Hobbs, AJ 129, 1993-2006, April 2005. URL http://www.atnf.csiro.au/research/pulsar/psrcat.

[62] D. A. Green, Bull. Astron. Soc. Ind. 37, 45-+, March 2009. URL http://www.mrao.cam.ac.uk/surveys/snrs/.

[63] M. A. Malkov and L. O'C Drury, Reports on Progress in Physics 64, 429-481, April 2001.

[64] L. O’C. Drury, ArXiv e-prints, September 2010 [arXiv: 1009.4799 ].

[65] P. Blasi and E. Amato, ArXiv e-prints, July 2010 [arXiv: 1007.4745$].$

[66] V. Tatischeff, $A \& A$ 499, 191-213, May 2009.

[67] A. Shalchi, Nonlinear Cosmic Ray Diffusion Theories, Springer, 2009.

[68] J. Lavalle, C. Bœhm, and J. Barthès, JCAP 2, 5-+, February 2010.

[69] J. Lavalle, Phys. Rev. D 82 (8), 083521-+, October 2010.

[70] J. A. Tauber, N. Mandolesi, J.-L. Puget et al., A\&A 520, A1+, September 2010.

[71] C. M. Walmsley, C. Bertout, F. Combes et al., A\&A 521, October 2010. 